\title{
Surfactin-based nanoparticles loaded with
} doxorubicin to overcome multidrug resistance in cancers

This article was published in the following Dove Press journal:

International Journal of Nanomedicine

\author{
Wenjing Huang' \\ Yan Lang' \\ Abdul Hakeem' \\ Yan Lei ${ }^{2}$ \\ Lu Gan' \\ Xiangliang Yang' \\ 'Department of Nanomedicine \\ and Biopharmaceuticals, National \\ Engineering Research Center for \\ Nanomedicine, College of Life \\ Science and Technology, Huazhong \\ University of Science and Technology, \\ Wuhan 430074, China; ${ }^{2}$ Pharmacy of \\ School Hospital, Huazhong University \\ of Science and Technology, Wuhan \\ 430074, China
}

Background: Multidrug resistance (MDR) is one of the major obstacles to successful cancer chemotherapy. Developing efficient strategies to reverse MDR remains a major challenge. Surfactin (SUR), a cyclic lipopeptide biosurfactant, has been found to display anticancer activity.

Methods: In this paper, SUR was assembled by solvent-emulsion method to load the anticancer drug doxorubicin (DOX). The cytotoxicity of DOX-loaded SUR nanoparticles (DOX@SUR) against DOX-resistant human breast cancer MCF-7/ADR is measured by MTT assay. The cellular uptake and intracellular retention of DOX@SUR are determined by flow cytometry. The tumor accumulation and anticancer activity of DOX@SUR are evaluated in MCF-7/ADRbearing nude mice.

Results: DOX@SUR induce stronger cytotoxicity against DOX-resistant human breast cancer MCF-7/ADR cells compared to free DOX. DOX@SUR nanoparticles exhibit enhanced cellular uptake and decreased cellular efflux, which might be associated with reduced P-glycoprotein expression. After internalization into MCF-7/ADR cells by macropinocytosis- and caveolinmediated endocytosis, DOX@SUR nanoparticles are colocalized with the lysosomes and translocated to the nucleus to exert cytotoxicity. Furthermore, in vivo animal experiment shows that the DOX@SUR nanoparticles are accumulated more efficiently in tumors than free DOX. Meanwhile,DOX@SUR nanoparticles display stronger tumor inhibition activity and fewer side effects in MCF-7/ADR-bearing nude mice.

Conclusion: This study indicates that SUR-based nanocarrier might present a promising platform to reverse MDR in cancer chemotherapy.

Keywords: multidrug resistance, cellular uptake, cellular efflux, biodistribution

\section{Introduction}

Breast cancer is the leading cause of cancer mortality in women worldwide. ${ }^{1}$ Doxorubicin (DOX) is widely used as a chemotherapeutic agent in the clinical therapy of breast cancer patients. ${ }^{2-4}$ Unfortunately, multidrug resistance (MDR) is considered as one of the primary impediments to successful cancer chemotherapy. ${ }^{5}$ Therefore, exploring how to overcome MDR is extremely crucial for breast cancer treatment. The most common mechanism of MDR is correlated with overexpression of ATPbinding cassette transporters, particularly P-glycoprotein (P-gp), leading to the efflux of many anticancer drugs. ${ }^{6}$ Nanoparticle-based therapeutics have been considered as one of the most prospective platforms in drug delivery applications due to increased drug accumulation in solid tumor by enhanced permeability and retention (EPR) and MDR reversal through bypassing or inhibiting P-gp activity. ${ }^{7-9}$
Correspondence: Lu Gan National Engineering Research Center for Nanomedicine, College of Life Science and Technology, Huazhong University of Science and Technology, Wuhan 430074, China

Tel +86 2787792147

Fax +86 2787792234

Email lugan@mail.hust.edu.cn 
Surfactin (SUR) is a cyclic lipopeptide biosurfactant containing heptapeptide (LLDLLDL) and a beta-hydroxy fatty acid produced by Bacillus subtilis. ${ }^{10}$ In addition to functioning as a biosurfactant owing to its amphipathic property, SUR has been found to exhibit versatile bioactive features including antibacterial, ${ }^{11,12}$ antiviral, ${ }^{13}$ anti-inflammatory,,${ }^{14,15}$ antiproliferative, ${ }^{16}$ adjuvant for immunization and antitumor properties. ${ }^{17-22}$ Recently, SUR has been found to inhibit the proliferation of MCF-7 cells by inducing apoptosis through a ROS/JNK-mediated mitochondrial/caspase pathway and decrease the drug efflux capacity of P-gp by reducing the function of ATP ${ }^{18}$ Based on the unique amphipathic properties, SUR has the potential to self-assemble into nanoparticles at a certain condition to function as a drug carrier for loading hydrophobic drugs. ${ }^{23}$ Combining the anticancer activity of SUR and the characteristics of nanoparticles such as EPR effects and MDR reversal, it might be promising to improve cancer chemotherapy by designing SUR as a carrier to load anticancer drugs.

Herein, we explore the unique ability of SUR nanoparticles as an anticancer drug carrier to overcome MDR. DOXloaded SUR nanoparticles (DOX@SUR) were synthesized by a solvent-emulsion method. The in vitro cytotoxicity and related mechanism to overcome MDR were studied in DOXsensitive human breast cancer MCF-7 and DOX-resistant MCF-7/ADR. The therapeutic outcomes were further examined in MCF-7/ADR xenograft model. The rational design of drug carriers based on SUR might have great significance in achieving efficient cancer therapy.

\section{Materials and methods}

\section{Materials}

Doxorubicin hydrochloride $(\mathrm{DOX} \cdot \mathrm{HCl})$ was provided by Beijing Huafeng United Technology (Beijing, China). SUR was purchased from Sigma Aldrich (St Louis, MO, USA). RPMI 1640 medium, penicillin-streptomycin and trypsin without EDTA were obtained from Thermo Fisher Scientific (Waltham, MA, USA). Fetal bovine serum was procured from Thermo Fisher Scientific. 4,6-Diamidino2-phenylindole and MTT were purchased from Biosharp (Anhui, China). LysoTracker Green was obtained from Thermo Fisher Scientific. All other reagents were of analytical grade and used without further purification.

\section{Synthesis of DOX@SUR nanoparticles}

DOX@SUR nanoparticles were synthesized by a solventemulsion method. Briefly, DOX $\cdot \mathrm{HCl}$ aqueous solution $(1.0 \mathrm{mg} / \mathrm{mL})$ was mixed with excess triethylamine $(15 \mu \mathrm{L} / \mathrm{mL}$ solution) in chloroform for $40 \mathrm{~min}$ to obtain hydrophobic
DOX. Then, this mixture was dropped into SUR aqueous solution $(2 \mathrm{mg} / \mathrm{mL})$ under ultrasonic emulsification for $5 \mathrm{~min}$, followed by constant stirring at $40^{\circ} \mathrm{C}$ overnight to volatilize chloroform. DOX@SUR nanoparticles were purified in an ultrafilter tube (cutoff molecular weight 3000; EMD Millipore) by centrifugation at 4,000 rpm for $20 \mathrm{~min}$ to remove free DOX. The blank SUR nanoparticles were formed by the same method, except that no DOX was added. The drug loading capacity of SUR nanoparticles was calculated using the equation: drug loading capacity = $\left(\mathrm{W}_{\text {administered dose }}-\mathrm{W}_{\text {residual dose in solution }}\right) / \mathrm{W}_{\mathrm{SUR}} \times 100 \%$. The size distribution and zeta potential of the nanoparticles were determined by dynamic light scattering (Zetasizer Nano ZS90; Malvern Instruments Ltd, Worcestershire, UK). The surface morphology of nanoparticles was observed with a transmission electron microscope (Tecnai G2-20; FEI Corp., Eindhoven, the Netherlands).

\section{In vitro drug release from DOX@SUR nanoparticles}

The release of DOX from DOX@SUR nanoparticles was investigated by the dialysis bag method. ${ }^{24}$ Briefly, $1 \mathrm{~mL}$ of $200 \mu \mathrm{g} / \mathrm{mL}$ DOX@SUR nanoparticles was placed in a dialysis bag (the cutoff molecular weight was 3000) and submerged fully into $25 \mathrm{~mL}$ of PBS, followed by stirring at $100 \mathrm{rpm}$ speed at $37^{\circ} \mathrm{C}$. Then, $1 \mathrm{~mL}$ of the release medium was taken out at the designated time points and replenished with the same volume of fresh PBS. The amount of DOX released was determined with a fluorescence spectrometer at Ex 488 nm/Em $560 \mathrm{~nm}$ using a DOX calibration curve.

\section{Cell culture and animals}

Sensitive human breast cancer MCF-7 cells and adriamycinresistant human breast cancer MCF-7 cells (MCF-7/ADR) were kindly provided by Dr Zhiping Zhang from the School of Pharmacy, Huazhong University of Science and Technology, Wuhan, China. MCF-7 and MCF-7/ADR cells were cultivated in RPMI 1640 medium containing 10\% fetal bovine serum, $100 \mathrm{U} / \mathrm{mL}$ penicillin G sodium and $100 \mu \mathrm{g} / \mathrm{mL}$ streptomycin sulfate at $37^{\circ} \mathrm{C}$ in a humidified $5 \% \mathrm{CO}_{2}$ incubator, except that $1 \mathrm{mg} / \mathrm{mL}$ DOX was added during MCF-7/MDR culture. We obtained the approval of the ethical committee of Huazhong University of Science and Technology for conducting research using these cells.

Female nude mice were purchased from HUAFUKANG Bioscience Co., Ltd (Beijing, China). All animals received care following the guidelines outlined in the "Guide for the Care and Use of Laboratory Animals". The mice were subcutaneously injected with $100 \mu \mathrm{L}$ of cell suspension $\left(2 \times 10^{6}\right.$ 
MCF-7/ADR cells per mouse) to initiate the formation of breast tumor. All animal experiments were approved and executed under the directions of the ethical committee of Huazhong University of Science and Technology.

\section{In vitro cytotoxicity of DOX@SUR nanoparticles}

The in vitro cytotoxicity of DOX@SUR nanoparticles was determined by MTT assay. Briefly, MCF-7 or MCF-7/ADR cells were initially cultured in complete RPMI 1640 medium for $24 \mathrm{~h}$. After rinsing with PBS, cells were incubated with blank SUR nanoparticles, free DOX or DOX@SUR nanoparticles at different concentrations of DOX. At the designated time points, cells were washed with PBS and then further incubated in a medium containing MTT solution $(5 \mathrm{mg} / \mathrm{mL})$ at $37^{\circ} \mathrm{C}$ for another $4 \mathrm{~h}$. Subsequently, the MTT solution containing medium was discarded and $150 \mu \mathrm{L}$ dimethyl sulfoxide was added to liquefy formazan crystals. The absorbance of formazan at $492 \mathrm{~nm}$ was measured using an iEMS microplate reader (Labsystems, Helsinki, Finland). ${ }^{25-27}$

\section{$\mathrm{Ki}-67, \mathrm{Bax}$ and $\mathrm{Bcl}-2$ expression in MCF-7/ADR cells}

MCF-7/ADR cells were treated with blank SUR nanoparticles, free DOX or DOX@SUR nanoparticles at a DOX concentration of $5 \mu \mathrm{g} / \mathrm{mL}$. At $12 \mathrm{~h}$ after treatment, the cells were harvested and total RNAs were extracted by using Trizol $^{\circledR}$ reagent (Thermo Fisher Scientific). Total RNA content was detected using a NanoDrop spectrophotometer (Thermo Fisher Scientific). cDNAs were generated by reverse transcription-polymerase chain reaction (PCR) method with the total RNA $(1 \mu \mathrm{g})$ from each sample. cDNA $(1 \mu \mathrm{g})$ was added to mixture containing ultrapure water $(4 \mu \mathrm{L})$, SYBR Green ( $4 \mu \mathrm{L}$; Takara, Tokyo, Japan), $10 \mu \mathrm{M}$ forward primer $(0.5 \mu \mathrm{L})$ and $10 \mu \mathrm{M}$ reverse primer $(0.5 \mu \mathrm{L})$. Sequences of Bcl-2, Bax, Ki-67 and glyceraldehyde 3-phosphate dehydrogenase primers used for real-time PCR are shown in Table S1. Quantitative real-time PCR was performed by using StepOnePlus ${ }^{\mathrm{TM}}$ Real-Time PCR (Thermo Fisher Scientific). Glyceraldehyde 3-phosphate dehydrogenase was considered as the internal reference, and $2^{-\Delta \Delta \mathrm{Ct}}$ was used to calculate the relative expression of target genes. ${ }^{26}$

\section{Cellular uptake of DOX@SUR nanoparticles}

MCF-7/ADR cells were cultured in 12-well plates at a density of $2 \times 10^{5}$ cells per well. After $24 \mathrm{~h}$ of incubation, the cells were treated with free DOX or DOX@SUR nanoparticles at $5 \mu \mathrm{g} / \mathrm{mL}$ DOX concentration for various time periods. Subsequently, the cells were washed twice with PBS, trypsinized and then resuspended in $400 \mu \mathrm{L}$ PBS for DOX fluorescence intensity measurement by flow cytometry (FC500; Beckman Coulter, Fullerton, CA, USA).

\section{Intracellular retention of DOX@SUR nanoparticles}

MCF-7/ADR cells were incubated in six-well plates at $1 \times 10^{5}$ cells per well. The cells were washed twice with PBS and incubated with free DOX or DOX@SUR nanoparticles at $5 \mu \mathrm{g} / \mathrm{mL}$ DOX concentration for $12 \mathrm{~h}$. Subsequently, the cells were washed twice with PBS and further incubated in blank medium for different time periods at $37^{\circ} \mathrm{C}$. The cells were washed twice with $\mathrm{PBS}$, trypsinized and then resuspended in $400 \mu \mathrm{L}$ PBS for DOX fluorescence intensity measurement by flow cytometry.

\section{P-gp expression}

MCF-7/ADR cells were cultured in 12-well plates at a density of $2 \times 10^{5}$ cells per well. After $12 \mathrm{~h}$ of incubation, the cells were treated with free DOX or DOX@SUR nanoparticles at $5 \mu \mathrm{g} / \mathrm{mL}$ DOX concentration for various time periods. Subsequently, the cells were washed twice with PBS, resuspended in $400 \mu \mathrm{L}$ PBS and then incubated with $20 \mu \mathrm{L}$ fluorescein isothiocyanate-conjugated mouse anti-human P-gp antibody (Becton, Dickinson and Company, Franklin Lakes, NJ, USA) for $15 \mathrm{~min}$. The fluorescence intensity of fluorescein isothiocyanate was measured by flow cytometry.

\section{Endocytic pathway of DOX@SUR nanoparticles}

MCF-7/ADR cells were preincubated in serum-free RPMI 1640 medium containing $2 \mathrm{mM}$ amiloride, $10 \mu \mathrm{g} / \mathrm{mL}$ chlorpromazine, $10 \mu \mathrm{g} / \mathrm{mL}$ filipin, $20 \mu \mathrm{g} / \mathrm{mL}$ nystain or $5 \mathrm{mM}$ methyl- $\beta$-cyclodextrin $(\mathrm{M} \beta \mathrm{CD})$ for $30 \mathrm{~min}$, respectively. ${ }^{25}$ The media were then changed to fresh serum-free medium containing the inhibitors and DOX@SUR nanoparticles at $5 \mu \mathrm{g} / \mathrm{mL}$ DOX concentration and incubated for another $1 \mathrm{~h}$. The cells were washed with PBS, harvested and then intracellular DOX fluorescence was determined by flow cytometry.

\section{Intracellular distribution of DOX@SUR nanoparticles}

MCF-7 or MCF-7/ADR cells were treated with DOX@ SUR nanoparticles at $5 \mu \mathrm{g} / \mathrm{mL}$ DOX concentration. At the designated time points, the cells were washed with PBS and then labeled with $5 \mu \mathrm{g} / \mathrm{mL}$ 4,6-diamidino-2-phenylindole or 
$200 \mathrm{nM}$ LysoTracker Green at $37^{\circ} \mathrm{C}$ for $20 \mathrm{~min}$. The cells were washed with PBS, fixed with $4 \%$ paraformaldehyde and then visualized by FV 1000 confocal microscope (Olympus Corporation, Tokyo, Japan).

\section{In vivo biodistribution of DOX@SUR nanoparticles}

MCF-7/ADR tumor-bearing nude mice were intravenously administered free DOX or DOX@SUR nanoparticles at $5 \mathrm{mg} \mathrm{DOX} / \mathrm{kg}$ dose. After 24 and $48 \mathrm{~h}$ injection, major organs including heart, liver, spleen, lung, kidney and tumor were resected and then imaged to assess ex vivo biodistribution of DOX@SUR in mice by in vivo fluorescence imaging (Thermo Fisher Scientific). These tissues were then washed with saline and homogenized with methanol. DOX content was measured by fluorescence spectrometer at Ex 488 nm/Em 560 nm.

\section{In vivo antitumor efficacy of DOX@SUR nanoparticles}

MCF-7/ADR tumor-bearing nude mice were intravenously administered saline, blank SUR nanoparticles, free DOX or DOX@SUR nanoparticles at a DOX dose of $5 \mathrm{mg} / \mathrm{kg}$ on day 1, 5 and 9. Tumor sizes were monitored with a digital caliper every day and calculated based on the formula: $\left(\mathrm{L} \times \mathrm{W}^{2}\right) / 2$, where $\mathrm{L}$ and $\mathrm{W}$ are the length and width of the tumor, respectively. On day 12, the mice were sacrificed, and the major organs were excised, fixed and sliced for H\&E staining.

\section{Statistical analysis}

All experiments were performed with at least three replicates. Student's $t$-test was used to carry out statistical analyses. Only $P<0.05$ was considered significant.

\section{Results and discussion}

\section{Synthesis and characterization of DOX@ SUR nanoparticles}

SUR is an attractive and powerful biosurfactant because of its special amphiphilic character (Figure 1A). When a hydrophobic organic solution of DOX (an anticancer drug model) was mixed with an aqueous solution of SUR, an unstable polymer emulsion was formed. With the evaporation of organic solvents, DOX was forced into the hydrophobic core of SUR nanoparticles, which resulted in the formation of a core-shell structure termed as DOX@SUR nanoparticles. Different feeding ratios of SUR and DOX were used to optimize the conditions of forming DOX@SUR nanoparticles (Table 1). The drug loading efficiency of SUR nanoparticles
A

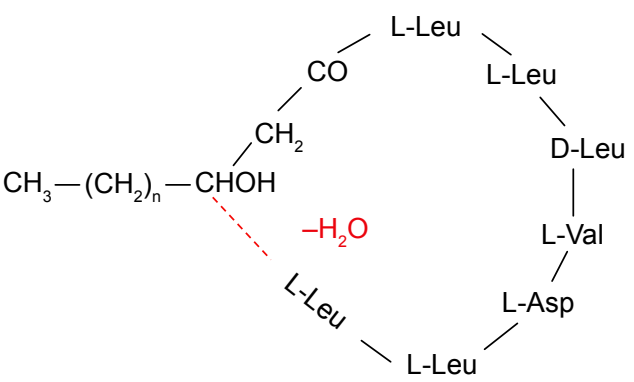

C

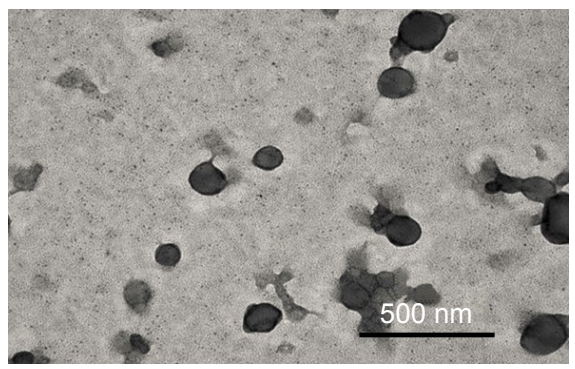

B

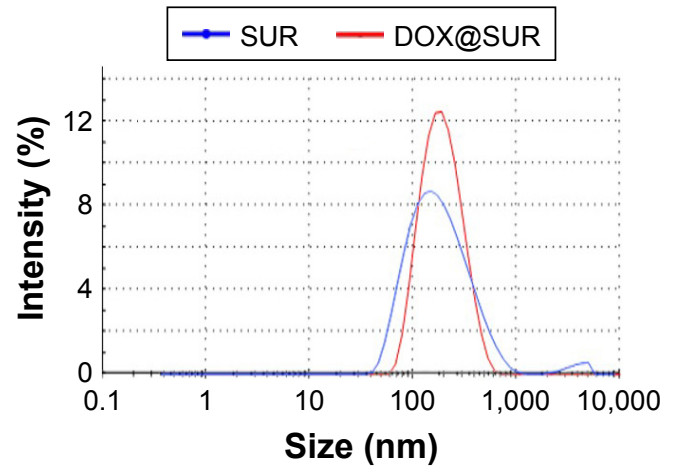

D

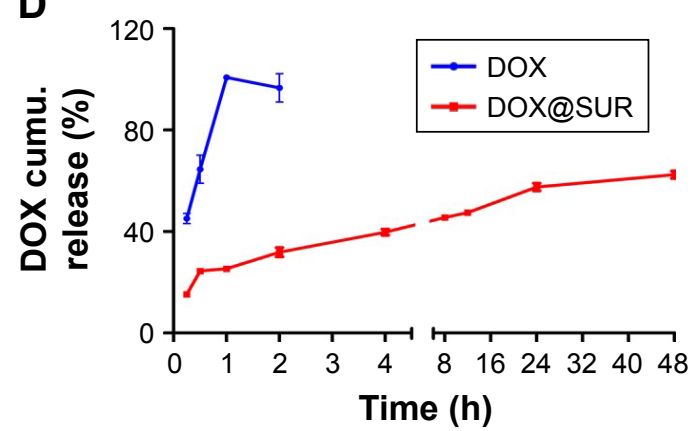

Figure I Characterization of DOX@SUR nanoparticles.

Notes: (A) Chemical structures of SUR. (B) Hydrodynamic diameter of blank SUR nanoparticles and DOX@SUR nanoparticles by DLS. (C) TEM image of DOX@SUR nanoparticles. (D) Drug release profiles of free DOX and DOX@SUR nanoparticles in PBS buffer (pH 7.4).

Abbreviations: DLS, dynamic light scattering; DOX, doxorubicin; SUR, surfactin; TEM, transmission electron microscope; DOX@SUR, DOX-loaded surfactin. 
Table I Characterization of DOX@SUR nanoparticles at different DOX/SUR feeding ratios

\begin{tabular}{lllll}
\hline $\begin{array}{l}\text { SUR:DOX } \\
\text { (mass ratio) }\end{array}$ & $\begin{array}{l}\text { Diameter } \\
(\mathbf{n m})\end{array}$ & $\begin{array}{l}\text { Zeta } \\
(\mathbf{m V})\end{array}$ & PDI & $\begin{array}{l}\text { Drug-loading } \\
\text { rate (\%) }\end{array}$ \\
\hline $5: 1$ & $249.5 \pm 49.5$ & $-32.5(0.4)$ & 0.133 & $10.6 \pm 0.5$ \\
$2: 1$ & $217.1 \pm 10.8$ & $-35.7(0.6)$ & 0.152 & $22.4 \pm 0.6$ \\
I:I & $160.2 \pm 10.2$ & $-36.1(0.5)$ & 0.138 & $25.8 \pm 0.5$ \\
I:2 & $207.1 \pm 00.4$ & $-36.6(0.6)$ & 0.221 & $27.6 \pm 7.8$ \\
\hline
\end{tabular}

Abbreviations: DOX, doxorubicin; PDI, polydispersity index; SUR, surfactin; DOX@SUR, DOX-loaded surfactin.

increased with increasing amounts of DOX input and reached $25.8 \%$ at the SUR/DOX feeding ratio of 1 . The size of the DOX@SUR nanoparticles formed became smaller at a certain range of feeding ratio (SUR:DOX $\leq 1: 1$ ). The size ofDOX@SUR nanoparticles was the smallest at the SUR/ DOX feeding ratio of 1 . Considering that the drug loading efficiency increased slowly when the SUR/DOX feeding ratio was $<1$, we formed DOX@SUR nanoparticles at the feeding ratio of 1 in further experiments.

Dynamic light scattering showed the size and zeta potential of DOX@SUR nanoparticles to be about $160 \mathrm{~nm}$ (Figure 1B) and $-36.0 \mathrm{mV}$, respectively. Transmission electron microscopic imaging showed that DOX@SUR was monodisperse and nearly spherical (Figure 1C). DOX release profile analysis from DOX@SUR nanoparticles showed that about 66.5\% DOX was released from DOX@SUR even after $48 \mathrm{~h}$ of incubation in PBS (Figure 1D). However, free DOX exhibited a burst release during $1 \mathrm{~h}$ of incubation. These results indicated that DOX@SUR nanoparticles displayed a slow drug release capacity.

\section{In vitro cytotoxicity of DOX@SUR nanoparticles}

To determine the biological function of DOX@SUR nanoparticles, the in vitro cytotoxicity against MCF-7 and MCF-7/ ADR cells was explored by MTT assay. As expected, blank SUR nanoparticles, free DOX and DOX@SUR nanoparticles displayed a dose-dependent cytotoxicity against both MCF-7 cells and MCF-7/ADR cells. However, no obvious difference in cytotoxicity of free DOX and DOX@SUR nanoparticles at a low DOX concentration was observed in MCF-7 cells, and the cytotoxicity of free DOX at $1.2 \mu \mathrm{g} / \mathrm{mL}$ concentration was stronger than that of DOX@SUR (Figure 2A).
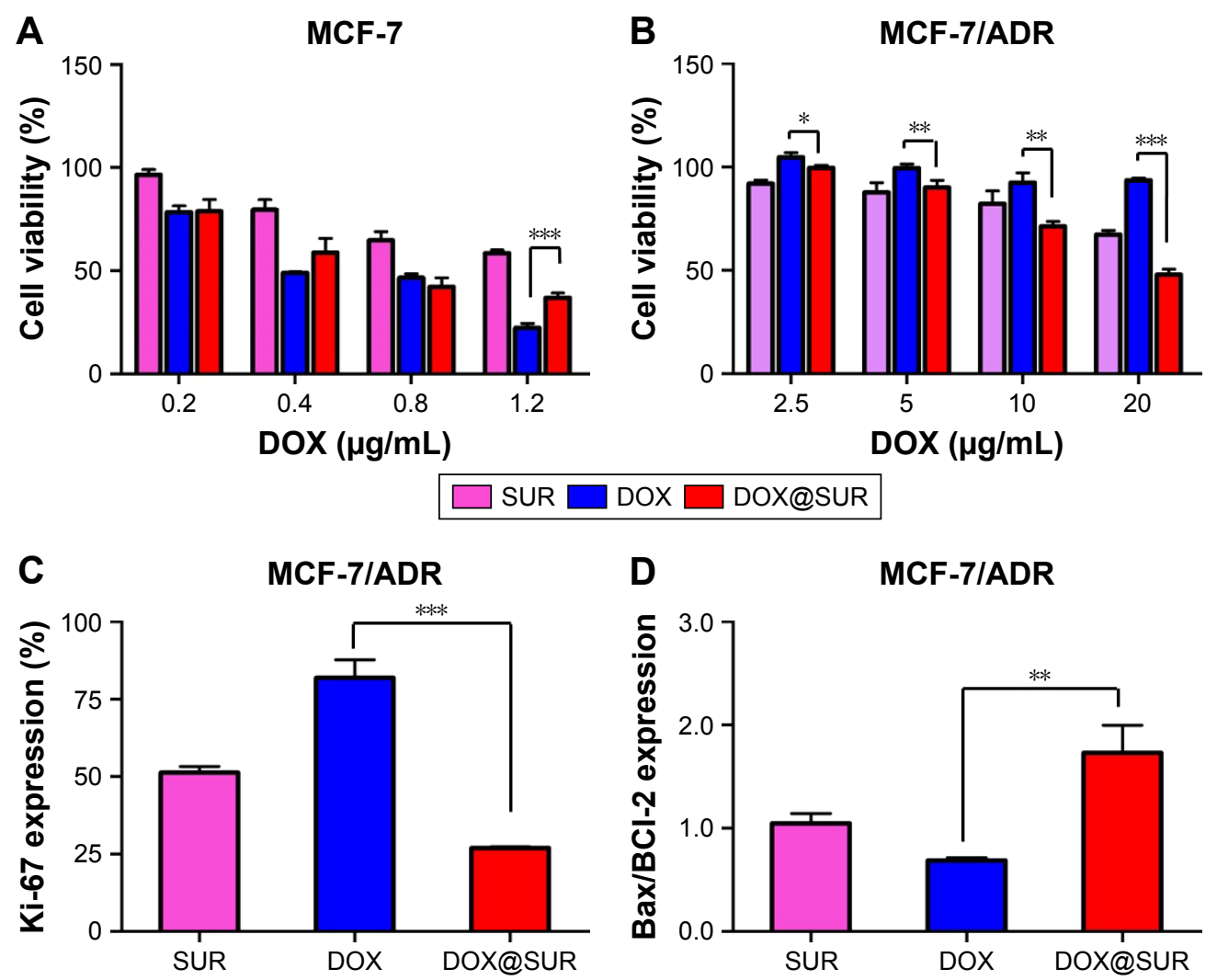

Figure 2 MDR reversal capacity of DOX@SUR nanoparticles.

Notes: (A, B) Cytotoxicity against (A) MCF-7 and (B) MCF-7/ADR cells after treatment with blank SUR nanoparticles, free DOX or DOX@SUR nanoparticles at different DOX concentrations for $48 \mathrm{~h}$ by MTT assay. (C, D) The mRNA expression of (C) Ki-67 and (D) Bax/Bcl-2 in MCF-7/ADR cells treated with blank SUR nanoparticles, free DOX or DOX@SUR nanoparticles at a DOX concentration of $5 \mu \mathrm{g} / \mathrm{mL}$ for $12 \mathrm{~h}$ by real-time RT-PCR. Data are expressed as mean value \pm SD ( $\mathrm{n}=3$ ). $* P<0.05$, $* * P<0.01$, *** $P<0.001$.

Abbreviations: DOX, doxorubicin; MDR, multidrug resistance; RT-PCR, reverse transcription-polymerase chain reaction; SUR, surfactin; DOX@SUR, DOX-loaded surfactin. 
This could be due to the reason that free DOX was easier to enter the cells than DOX@SUR in MCF-7 cells. However, in DOX-resistant MCF-7/ADR cells, DOX@SUR nanoparticles exhibited stronger cytotoxicity than free DOX (Figure 2B). Furthermore, we examined the $\mathrm{IC}_{50}$ value of free DOX and DOX@SUR nanoparticles (Table S2). The $\mathrm{IC}_{50}$ of free DOX in MCF-7/ADR cells was estimated to be $147 \mu \mathrm{g} / \mathrm{mL}$ after treatment for $48 \mathrm{~h}$, about 420 -fold of that in MCF-7 cells, confirming DOX resistance in MCF-7/ ADR cells. Consistently, there was no obvious difference in $\mathrm{IC}_{50}$ of free DOX and DOX@SUR nanoparticles in MCF-7 cells. In contrast, the $\mathrm{IC}_{50}$ of DOX@SUR nanoparticles decreased to $15.6 \mu \mathrm{g} / \mathrm{mL}$ in MCF-7/ADR cells, which was about $10.5 \%$ of that of free DOX, suggesting that DOX@ SUR nanoparticles efficiently reversed the drug resistance in MCF-7/ADR cells. To further confirm this, the expression of proliferation- and apoptosis-related genes was evaluated by real-time PCR. As shown in Figure 2C and D, treatment with DOX@SUR nanoparticles significantly decreased the expression of $\mathrm{Ki}-67$ and upregulated the ratio of $\mathrm{Bax} / \mathrm{Bcl}-2$ gene expression in MCF-7/ADR cells compared to that with free DOX, suggesting that DOX@SUR nanoparticles had stronger ability to inhibit proliferation and induce apoptosis in MCF-7/ADR cells.

\section{Cellular uptake and efflux of DOX@SUR nanoparticles}

To understand the underlying mechanism of DOX@SUR nanoparticles on MDR reversal in MCF-7/ADR cells, we initially examined their cellular uptake using flow cytometry. Both free DOX and DOX@SUR nanoparticles displayed enhanced cellular uptake by MCF-7 and MCF-7/ADR cells in a dose- and time-dependent manner (Figure 3A and B). Consistent with the stronger cytotoxicity of free DOX against MCF-7 cells (Figure 2A), more free DOX was internalized into MCF-7 cells than DOX@SUR nanoparticles (Figure 3A). In contrast, higher DOX fluorescence was detected in DOX@ SUR nanoparticle-treated MCF-7/ADR cells than that in free DOX-treated cells (Figure 3B), suggesting enhanced cellular uptake of DOX@SUR nanoparticles by MCF-7/ADR cells. The increased internalization of DOX@SUR nanoparticles into MCF-7/ADR cells was further confirmed by confocal laser scanning microscopy (Figure S1).

Besides enhanced cellular uptake, it is widely acknowledged that cancer MDR is often allied with drug efflux pumps overexpressed on the cell membrane, such as P-gp, to efflux the intracellular drug. ${ }^{14}$ To determine whether DOX@SUR nanoparticles decreased drug efflux, MCF-7/ADR cells were first incubated with free DOX or DOX@SUR for 4h, followed by incubation in fresh medium for various time intervals and then determining the intracellular DOX fluorescent intensity by flow cytometry (Figure 3C). The result showed that $62.2 \%$ of free DOX was eliminated from the MCF-7/ADR cells within $2 \mathrm{~h}$, but in contrast, only 38.4\% of the DOX carried by DOX@SUR nanoparticles was expelled even after 8 h, revealing that DOX@SUR nanoparticles could more effectively escape from the drug efflux action compared to free DOX. To determine if the reduced drug efflux of DOX@ SUR was attributed to P-gp expression change, P-gp protein expression in MCF-7/ADR cells treated with free DOX or DOX@SUR nanoparticles was determined by immunofluorescence staining (Figure 3D). The P-gp expression of MCF-7/ ADR cells increased after treatment with DOX, whereas the P-gp expression in DOX@SUR nanoparticle-treated cells was significantly lower than that in free DOX-treated cells, demonstrating that the successful retention of DOX@SUR nanoparticles might be attributed to P-gp suppression.

\section{Intracellular trafficking of DOX@SUR nanoparticles}

In view of the enhanced cellular uptake and decreased drug efflux of DOX@SUR nanoparticles in MCF-7/ADR cells, we further determined their endocytic pathway and intracellular trafficking. MCF-7/ADR cells were pretreated with various endocytic inhibitors, followed by treatment with DOX@SUR nanoparticles for $4 \mathrm{~h}$ and then the intracellular fluorescence of DOX@SUR nanoparticles was determined by flow cytometry. As shown in Figure 4A, amiloride (an inhibitor of macropinocytosis-dependent endocytosis) and nystain, filipin and $\beta$-cyclodextrin (caveolin-mediated endocytosis inhibitors) significantly inhibited the internalization of DOX@SUR nanoparticles, whereas chlorpromazine (an inhibitor of clathrin-mediated endocytosis) did not show any notable inhibition on the cellular uptake of DOX@SUR nanoparticles. The data suggested that macropinocytosis- and caveolin-mediated endocytosis might be involved in the cellular uptake of DOX@SUR nanoparticles.

To track the intracellular fate of DOX@SUR nanoparticles after internalization, confocal microscopy was used to determine the colocalization of DOX@SUR nanoparticles with lysosomes and nuclei. As shown in Figure 4B, after the MCF-7/ADR cells were treated with DOX@SUR nanoparticles for 2 h, the DOX@SUR nanoparticles were mostly colocalized with lysosomes and small amounts were found to colocalize with nuclei, demonstrating that the internalized DOX@SUR nanoparticles were mainly transmitted 


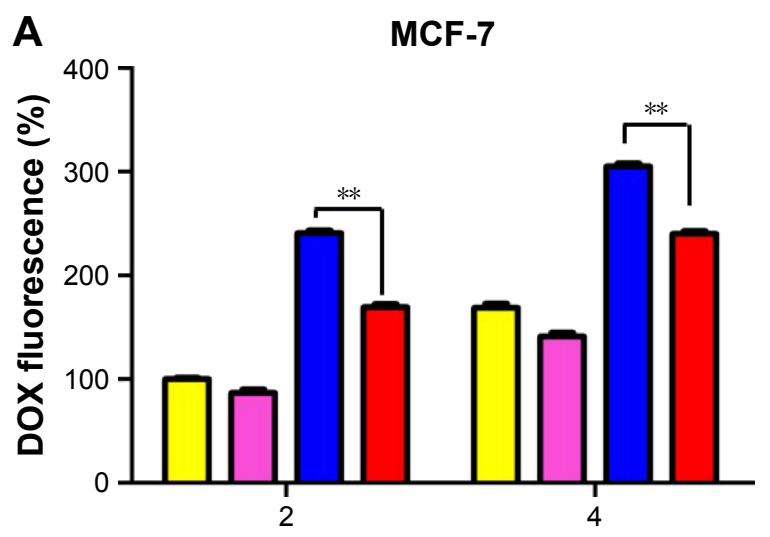

Time (h)
B MCF-7IADR

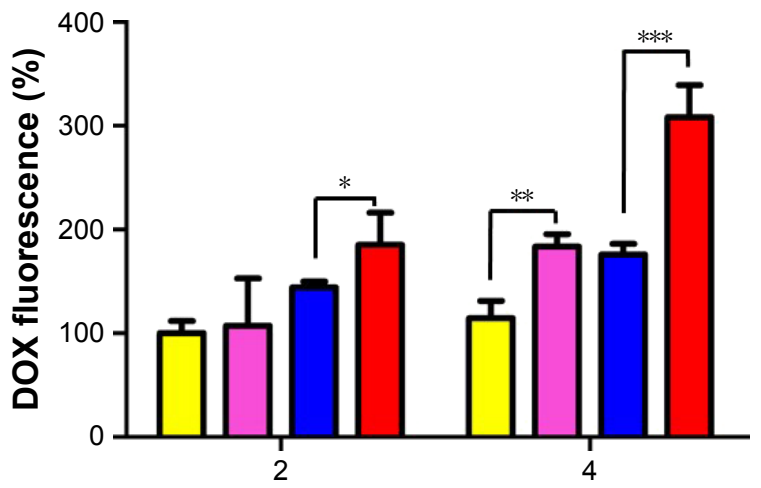

Time (h)

DOX $2.5 \mu \mathrm{g} \square$ DOX@SUR $2.5 \mu \mathrm{g} \square$ DOX $5 \mu \mathrm{g} \square$ DOX@SUR $5 \mu \mathrm{g}$
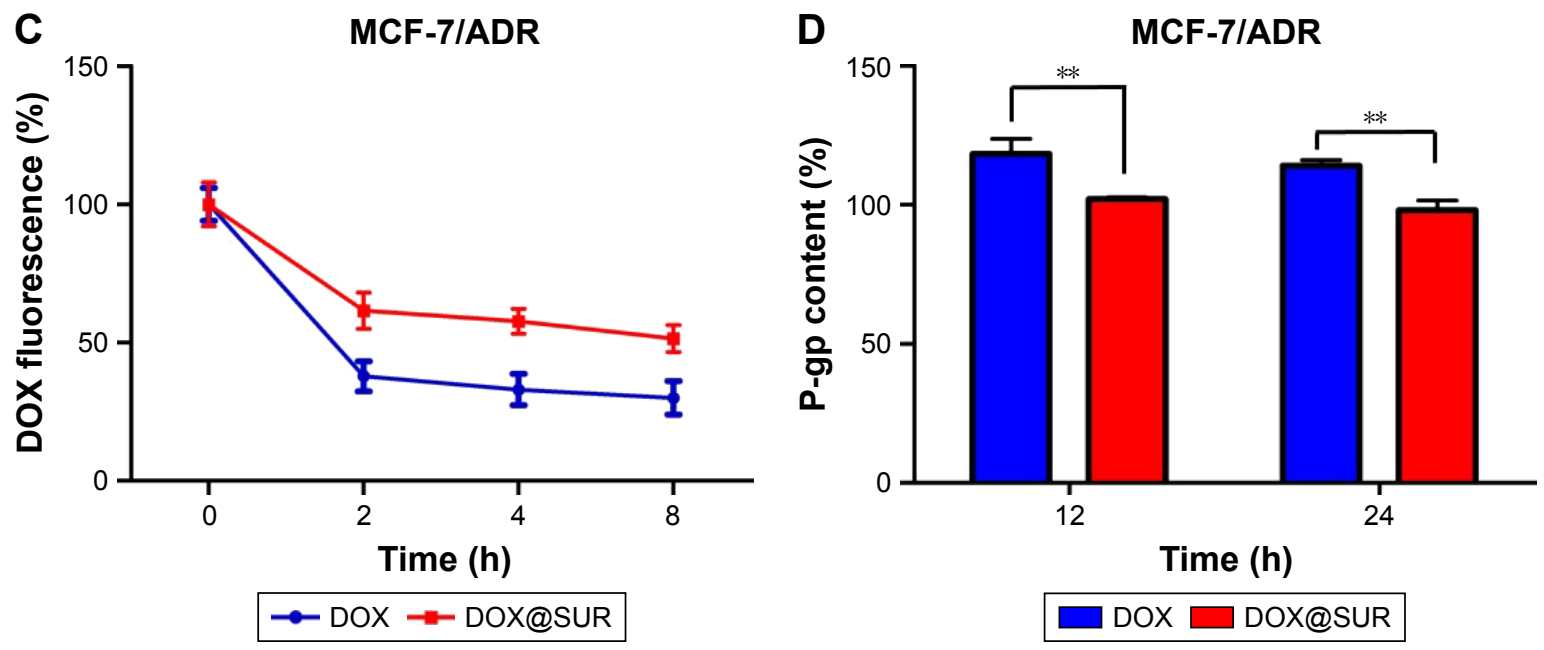

Figure 3 Cellular uptake and retention of DOX@SUR nanoparticles.

Notes: (A) Relative DOX fluorescence intensity in MCF-7 and (B) MCF-7/ADR cells after treatment with free DOX or DOX@SUR nanoparticles at different DOX concentrations for different time courses. (C) Relative DOX fluorescence intensity in MCF-7/ADR cells after pretreatment with free DOX or DOX@SUR nanoparticles at a DOX concentration of $5 \mu \mathrm{g} / \mathrm{mL}$ for $12 \mathrm{~h}$, followed by incubation in fresh medium for different time courses. (D) Relative P-gP expression in MCF-7/ADR cells after treatment with free DOX or DOX@SUR nanoparticles at a DOX concentration of $5 \mu \mathrm{g} / \mathrm{mL}$ for 12 or $24 \mathrm{~h}$ by flow cytometry. Data are expressed as mean value \pm SD ( $\mathrm{n}=3$ ). $* P<0.05, * * P<0.01$, $* * * P<0.001$.

Abbreviations: DOX, doxorubicin; P-gP, P-glycoprotein; SUR, surfactin; DOX@SUR, DOX-loaded surfactin.

to the lysosomes and released DOX to the nuclei to exert cytotoxicity. To further confirm the nuclear translocation of DOX@SUR nanoparticles, MCF-7/ADR cells were treated with DOX@SUR nanoparticles for different time courses (Figure S2). Consistently, more DOX@SUR nanoparticles were internalized into cells in a time-dependent manner. More importantly, more DOX was detected in the nuclei with increase of incubation time, revealing that DOX was released and translocated to the nuclei after the cellular uptake of DOX@SUR nanoparticles.

\section{In vivo biodistribution of DOX@SUR nanoparticles}

It is widely acknowledged that the in vivo biodistribution of anticancer drugs affects their therapeutic impact and potential side reactions. To detect the biodistribution of DOX@SUR nanoparticles, MCF-7/ADR-bearing nude mice were intravenously injected with free DOX or DOX@SUR nanoparticles. At $48 \mathrm{~h}$ after injection, DOX fluorescence in the major organs (including heart, liver, spleen, lung and kidney) and tumor was first examined by living fluorescence imaging (Figure 5A). DOX@SUR nanoparticles displayed stronger DOX fluorescence in the tumor than free DOX. In order to further confirm the enhanced tumor accumulation capacity of DOX@SUR nanoparticles, quantitative analysis of DOX in these major organs and tumors was conducted after 24 and $48 \mathrm{~h}$ of injection (Figure 5B). DOX content in the tumor tissues of mice treated with DOX@SUR nanoparticles was nearly 2.1-fold that of free DOX at $24 \mathrm{~h}$ and it even increased to 3.5 -fold after $48 \mathrm{~h}$ of injection. Moreover, DOX content in 


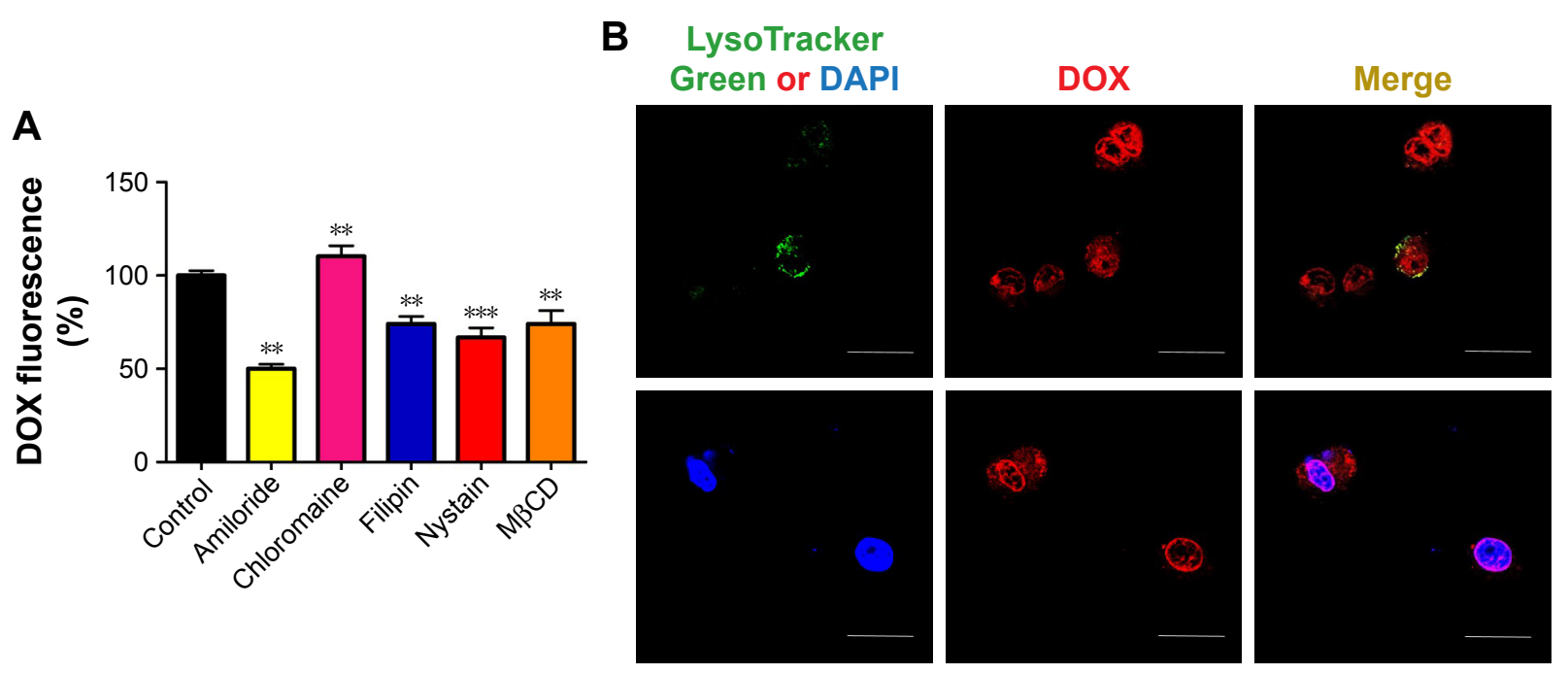

Figure 4 Endocytic pathway and intracellular trafficking of DOX@SUR nanoparticles.

Notes: (A) Relative DOX fluorescence intensity in MCF-7/ADR cells after pretreatment with $2 \mathrm{mM}$ amiloride, $10 \mu \mathrm{g} / \mathrm{mL}$ chlorpromazine, $10 \mu \mathrm{g} / \mathrm{mL}$ filipin, $20 \mu \mathrm{g} / \mathrm{mL}$ nystain or $5 \mathrm{mM} \mathrm{M \beta CD} \mathrm{for} 30 \mathrm{~min}$ and then treatment with DOX@SUR nanoparticles at a DOX concentration of $5 \mu \mathrm{g} / \mathrm{mL}$ for I h, respectively. Data are expressed as mean value $\pm S D(n=3)$. $* * P<0.01, * * * P<0.001$ compared to the control group. (B) Confocal microscopic images of the intracellular localization of DOX@SUR nanoparticles in MCF-7/ADR cells treated with DOX@SUR nanoparticles at a DOX concentration of $5 \mu \mathrm{g} / \mathrm{mL}$ for 4 h. Scale bar: $25 \mu \mathrm{m}$.

Abbreviations: DAPI, 4,6-diamidino-2-phenylindole; DOX, doxorubicin; SUR, surfactin; DOX@SUR, DOX-loaded surfactin; M $\beta C D$, methyl- $\beta$-cyclodextrin.

the heart of mice treated with DOX@SUR was slightly lower than that of mice treated with free DOX either after 24 or $48 \mathrm{~h}$, indicating that nanoparticles might have the ability to reduce DOX cardiotoxicity compared to free DOX. These facts consistently suggested that DOX@SUR nanoparticles exhibited good tumor-targeting efficiency, which was mostly due to their enhanced EPR effect.

\section{Antitumor activity of DOX@SUR nanoparticles}

The efficiency of DOX@SUR nanoparticles to overwhelm MDR was further evaluated in a drug-resistant MCF-7/ADR tumor model. The tumor growth curves (Figure 6A) displayed that blank SUR nanoparticles showed almost similar tumor growth inhibition activity with free DOX. DOX@ SUR nanoparticles exhibited the strongest suppression of tumor growth compared to free DOX and blank SUR nanoparticles. Moreover, the weights of excised tumors after treatment also showed a similar trend (Figure 6B and $\mathrm{C}$ ). These results indicate that DOX@SUR nanoparticles noticeably enhanced the anticancer potential of free DOX and SUR, which might be attributed to the enhanced tumor targeting and reversal MDR of DOX@ SUR nanoparticles.
A

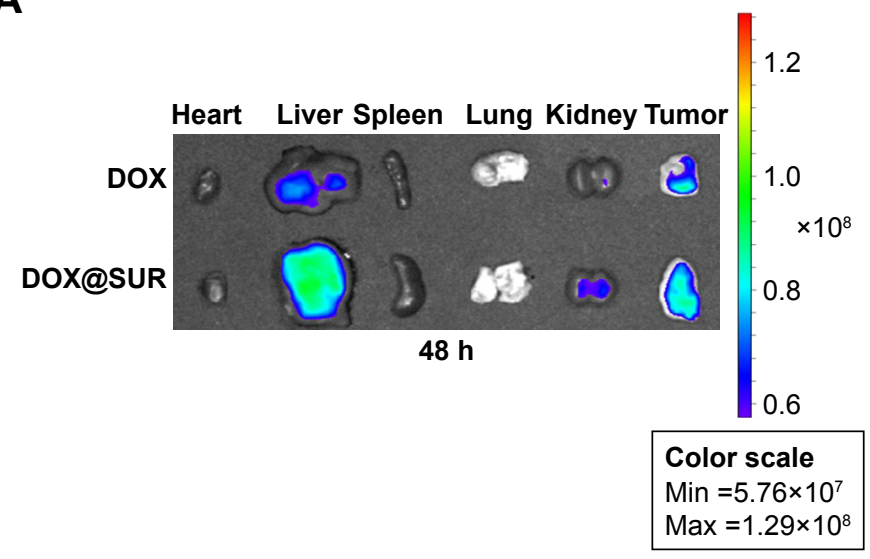

B

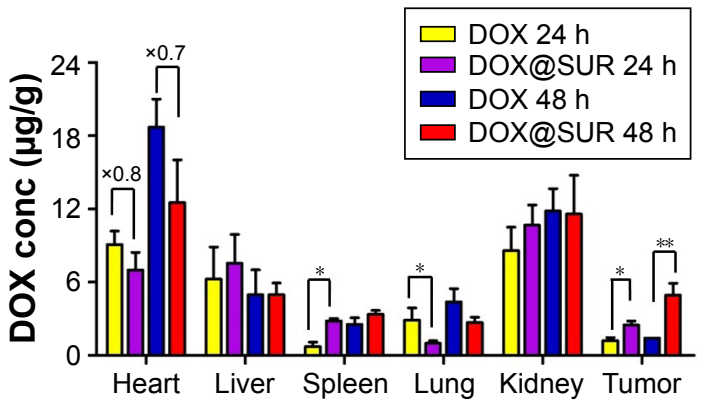

Figure 5 In vivo biodistribution of DOX@SUR nanoparticles.

Notes: (A) Ex vivo fluorescent images of DOX in major organs and tumors at $48 \mathrm{~h}$ after intravenous injection of free DOX or DOX@SUR nanoparticles at the DOX dosage of $5 \mathrm{mg} / \mathrm{kg}$, respectively. (B) Quantitative analysis of DOX distribution in the major organs and tumors at 24 and $48 \mathrm{~h}$ after intravenous injection of free DOX or DOX@SUR nanoparticles at the DOX dosage of $5 \mathrm{mg} / \mathrm{kg}$. Data are expressed as mean value $\pm S D(n=3) . * P<0.05, * * p<0.01$.

Abbreviations: DOX, doxorubicin; SUR, surfactin; DOX@SUR, DOX-loaded surfactin. 
A
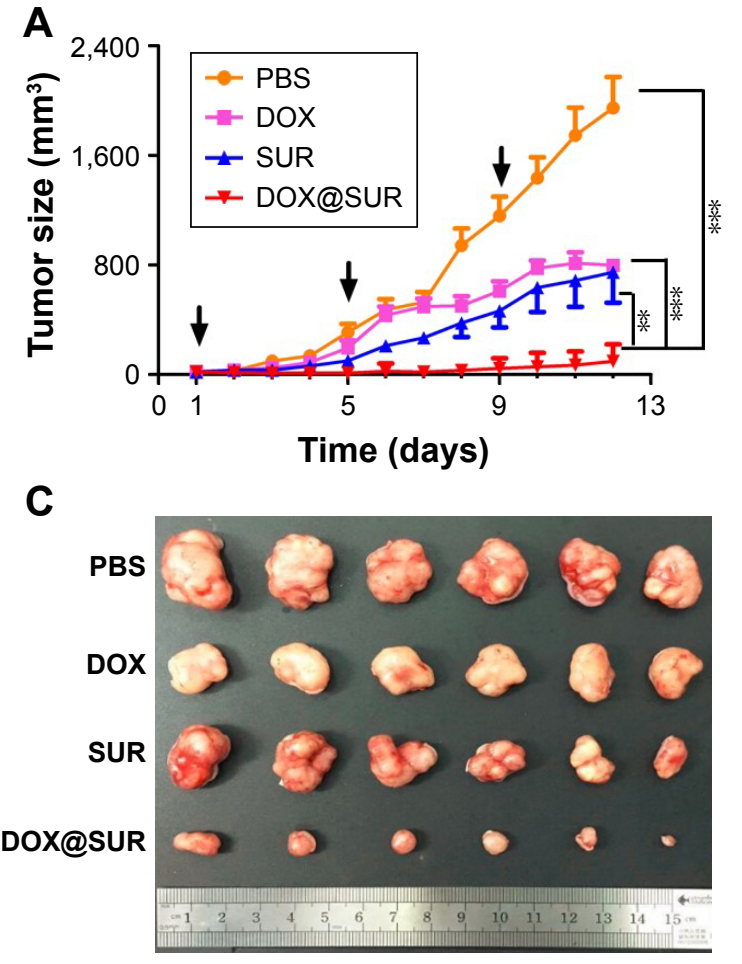
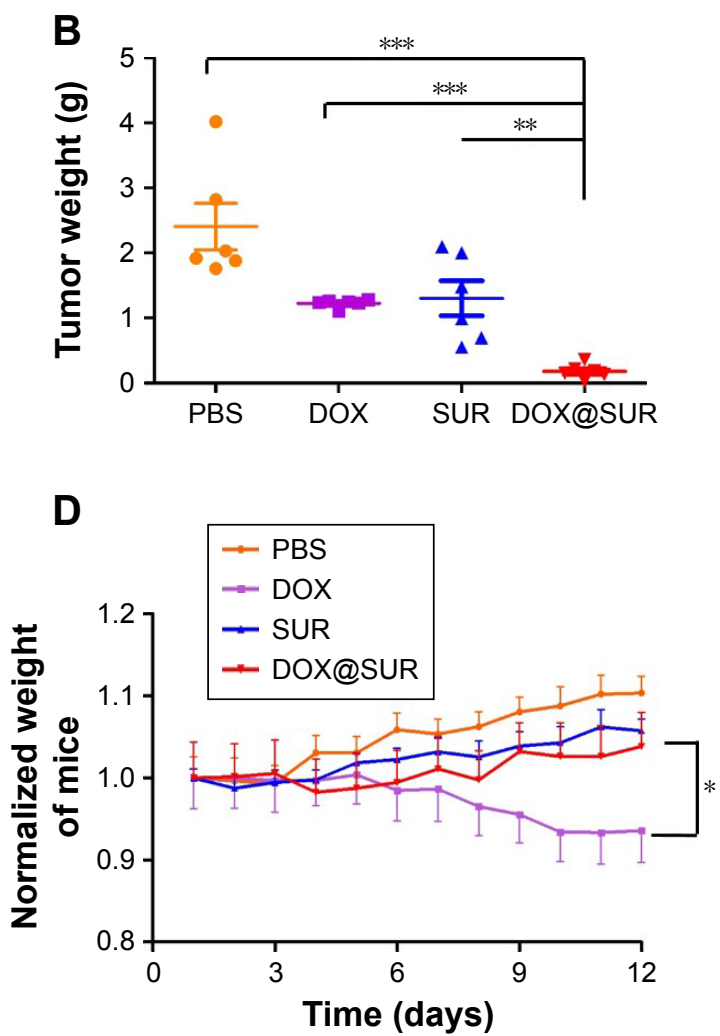

Figure 6 In vivo antitumor study of DOX@SUR nanoparticles in Balb/c nude mice implanted with MCF-7/ADR cells.

Notes: (A) Tumor growth inhibition profiles in MCF-7/ADR-bearing nude mice after intravenous injection of PBS, blank SUR nanoparticles, free DOX or DOX@SUR nanoparticles at the DOX dose of $5 \mathrm{mg} / \mathrm{kg}$. Black arrows indicate the injection time points. (B) Tumor weight at the end of treatment. (C) Photographs of the tumors at the end of treatment. (D) Body weight change of MCF-7/ADR-bearing nude mice during treatment. Data are expressed as mean value $\pm S D(n=6)$. $* P<0.05$, $* * P<0.01$, $* * * P<0.001$.

Abbreviations: DOX, doxorubicin; SUR, surfactin; DOX@SUR, DOX-loaded surfactin.

In addition to possessing potential activity of reversing MDR, it is equally important for the nanosized drug delivery systems to be biosafe in vivo. ${ }^{28}$ Body weight is considered as an important indicator of general systemic toxicity. ${ }^{29} \mathrm{The}$ body weight increased in PBS group and decreased in free DOX-treated group (Figure 6D). However, the body weight in DOX@SUR nanoparticle-treated group was substantially unchanged compared to that of PBS group. These findings indicate that the DOX@SUR nanoparticles did not show obvious toxicity, even though free DOX might exhibit systemic toxicity. Furthermore, histopathologic changes of major organs including heart, liver, spleen, lung and kidney were evaluated in MCF-7/ADR xenograft tumor by H\&E staining (Figure 7). Cardiotoxicity is the main threat of DOX-based chemotherapy. Noticeable neutrophil accumulation was seen in the heart slices of free DOXtreated group, while DOX-caused cardiocytotoxicity was markedly inhibited in DOX@SUR-treated group. The other tested tissue sections did not show any significant lesion. In addition, serological analysis showed that DOX@SUR nanoparticles did not cause significant toxicity to liver and kidney function (Figure S3). These fallouts confirmed that DOX@SUR nanoparticles were capable of overcoming MDR and exhibited stronger anticancer activity with reduced adverse effects.

\section{Conclusion}

We have successfully formed DOX-loaded SUR nanoparticles to reverse MDR in cancer therapy. SUR with anticancer activity was self-assembled into nanoparticles to load DOX with a high drug loading efficiency. DOX@SUR nanoparticles exhibited enhanced cellular uptake and decreased cellular efflux in MCF-7/ADR cells due to P-gp inhibition, which resulted in augmented cytotoxicity compared to free DOX. After the DOX@SUR nanoparticles were internalized into MCF-7/ADR cells by macropinocytosis- and caveolinmediated endocytosis, they were transported to the lysosomes and then translocated to the nucleus to exert cytotoxicity. Moreover, DOX@SUR nanoparticles displayed enhanced tumor accumulation. Therefore, superior in vivo tumor inhibition activity of DOX@SUR nanoparticles was observed in MCF-7/ADR-bearing nude mice with fewer side effects. 

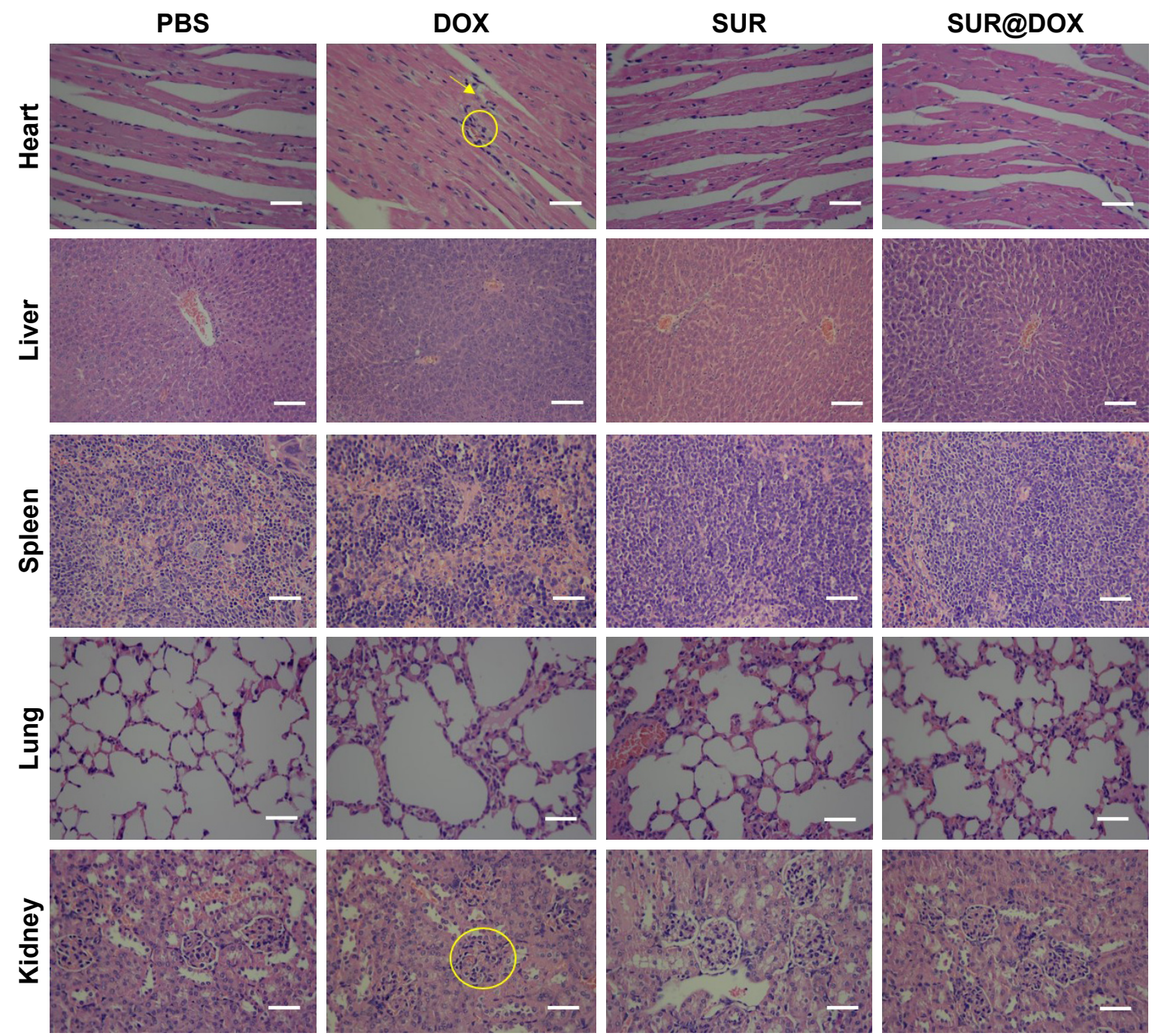

Figure $7 \mathrm{H} \& \mathrm{E}$ staining assay of major organs and tumors at the end of tumor growth inhibition studies.

Notes: Yellow arrows and rings in the heart and kidney sections of free DOX-treated mice indicate severe toxicity. Scale bar: $200 \mu \mathrm{m}$.

Abbreviations: DOX, doxorubicin; SUR, surfactin; DOX@SUR, DOX-loaded surfactin.

This study demonstrated that SUR-based nanoparticles might be used as potential anticancer drug carriers to reverse MDR in cancer chemotherapy.

\section{Acknowledgments}

This work was supported by National Basic Research Program of China (2015CB931802), National Natural Science Foundation of China (81672937, 81473171, 81773653 and 81627901) and Program for Changjiang Scholars and Innovative Research Team in University (IRT13016). We thank the Analytical and Testing Center of Huazhong University of Science and Technology for related analysis.

\section{Disclosure}

The authors report no conflicts of interest in this work.

\section{References}

1. Youlden DR, Cramb SM, Dunn NA, Muller JM, Pyke CM, Baade PD. The descriptive epidemiology of female breast cancer: an international comparison of screening, incidence, survival and mortality. Cancer Epidemiol. 2012;36(3):237-248.

2. Wei T, Liu J, Ma H, et al. Functionalized nanoscale micelles improve drug delivery for cancer therapy in vitro and in vivo. Nano Lett. 2013; 13(6):2528-2534.

3. Ren Y, Wang Y, Zhang Y, Wei D. Overcoming multidrug resistance in human carcinoma cells by an antisense oligodeoxynucleotide doxorubicin conjugate in vitro and in vivo. Mol Pharm. 2008;5(4):579-587.

4. Wang F, Wang YC, Dou S, Xiong MH, Sun TM, Wang J. Doxorubicintethered responsive gold nanoparticles facilitate intracellular drug delivery for overcoming multidrug resistance in cancer cells. ACS Nano. 2011;5(5):3679-3692.

5. Liu J, Ma H, Wei T, Liang $\mathrm{XJ}$. $\mathrm{CO}_{2}$ gas induced drug release from $\mathrm{pH}$-sensitive liposome to circumvent doxorubicin resistant cells. Chem Comm (Camb). 2012;48(40):4869-4871.

6. Klappe K, Hummel I, Hoekstra D, Kok JW. Lipid dependence of ABC transporter localization and function. Chem Phys Lipids. 2009; 161(2):57-64. 
7. Bao Y, Yin M, Hu X, et al. A safe, simple and efficient doxorubicin prodrug hybrid micelle for overcoming tumor multidrug resistance and targeting delivery. J Control Release. 2016;235:182-194.

8. Jiang L, Li L, He X, et al. Overcoming drug-resistant lung cancer by paclitaxel loaded dual-functional liposomes with mitochondria targeting and pH-response. Biomaterials. 2015;52:126-139.

9. Wang M, Thanou M. Targeting nanoparticles to cancer. Pharm Res. 2007;24(1):1029-1046.

10. Hwang MH, Lim JH, Yun HI, et al. Surfactin C inhibits the lipopolysaccharide-induced transcription of interleukin-1 $\beta$ and inducible nitric oxide synthase and nitric oxide production in murine RAW 264.7 cells. Biotechnol Lett. 2005;27(20):1605-1608.

11. Lee MH, Lee J, Nam YD, Lee JS, Seo MJ, Yi SH. Characterization of antimicrobial lipopeptides produced by Bacillus sp.LM7 isolated from chungkookjang, a Korean traditional fermented soybean food. Int J Food Microbiol. 2016;221:12-18.

12. Loiseau C, Schlusselhuber M, Bigot R, Bertaux J, Berjeaud JM, Verdon J. Surfactin from Bacillus subtilis displays an unexpected anti-Legionella activity. Appl Microbiol Biotechnol. 2015;99(12):5083-5093.

13. Kracht M, Rokos H, Ozel M, Kowall M, Pauli G, Vater J. Antiviral and hemolytic activities of surfactin isoforms and their methyl ester derivatives. J Antibiot (Tokyo). 1999;52(7):613-619.

14. Zhang Y, Liu C, Dong B, et al. Anti-inflammatory activity and mechanism of surfactin in lipopolysaccharide-activated macrophages. Inflammation. 2015;38(2):756-764.

15. Gan P, Jin D, Zhao X, et al. Bacillus-produced surfactin attenuates chronic inflammation in atherosclerotic lesions of ApoE(-/-) mice. Int Immunopharmacol. 2016;35:226-234.

16. Kim SY, Kim JY, Kim SH, et al. Surfactin from Bacillus subtilis displays anti-proliferative effect via apoptosis induction, cell cycle arrest and survival signaling suppression. FEBS Lett. 2007;581(5):865-871.

17. Vollenbroich D, Ozel M, Vater J, Kamp RM, Pauli G. Mechanism of inactivation of enveloped viruses by the biosurfactant surfactin from Bacillus subtilis. Biologicals. 1997;25(3):289-297.

18. Cao XH, Zhao SS, Liu DY, et al. ROS-Ca ${ }^{2+}$ is associated with mitochondria permeability transition pore involved in surfactin-induced MCF-7 cells apoptosis. Chem Biol Interact. 2011;190(1):16-27.
19. Cao XH, Liao ZY, Wang CL, Yang WY, Lu MF. Evaluation of a lipopeptide biosurfactant from Bacillus natto TK-1 as a potential source of anti-adhesive, antimicrobial and antitumor activities. Braz J Microbiol. 2009;40(2):373-379.

20. Cao XH, Liao ZY, Wang CL, et al. Purification and antitumour activity of a lipopeptide biosurfactant produced by Bacillus natto TK-1. Appl Biochem. 2009;52(Pt 2):97-106.

21. Christova N, Tuleva B, Kril A, et al. Chemical structure and in vitro antitumor activity of rhamnolipids from Pseudomonas aeruginosa BN10. Appl Biochem Biotechnol. 2013;170(3):676-689.

22. Cao XH, Wang AH, Wang CL, et al. Surfactin induces apoptosis in human breast cancer MCF-7 cells through a ROS/JNK-mediated mitochondrial/ caspase pathway. Chem Biol Interact. 2010;183(3):357-362.

23. Straus SK, Hancock RE. Mode of action of the new antibiotic for Grampositive pathogens daptomycin: comparison with cationic antimicrobial peptides and lipopeptides. Biochim Biophys Acta. 2006;1758(9): 1215-1223.

24. Yang H, Wang Q, Huang S, et al. Smart $\mathrm{pH} /$ redox dual-responsive nanogels for on-demand intracellular anticancer drug release. ACS Appl Mater Interfaces. 2016;8(12):7729-7738.

25. Xiao L, Xiong X, Sun X, et al. Role of cellular uptake in the reversal of multidrug resistance by PEG-b-PLA polymeric micelles. Biomaterials. 2011;32(22):5148-5157.

26. Zhang H, Liang C, Hou X, Wang L, Zhang D. Study of the combined treatment of lung cancer using gene-loaded immunomagnetic albumin nanospheres in vitro and in vivo. Int $J$ Nanomedicine. 2016;11: 1039-1050.

27. Lian $\mathrm{H}, \mathrm{Wu} \mathrm{J}, \mathrm{Hu} \mathrm{Y}$, Guo H. Self-assembled albumin nanoparticles for combination therapy in prostate cancer. Int J Nanomedicine. 2017; 12(1):7777-7787.

28. Liu J, Li Q, Zhang J, et al. Safe and effective reversal of cancer multidrug resistance using sericin-coated mesoporous silica nanoparticles for lysosome-targeting delivery in mice. Small. 2017;13(9):1602567.

29. Gupta B, Ramasamy T, Poudel BK, et al. Development of bioactive PEGylated nanostructured platforms for sequential delivery of doxorubicin and imatinib to overcome drug resistance in metastatic tumors. ACS Appl Mater Interfaces. 2017;9(11):9280-9290. 


\section{Supplementary materials}

Table SI Primer sequences used for real-time RT-PCR

\begin{tabular}{ll}
\hline Gene & Primers \\
\hline GADPH & Forward: GAGAGAAACCCGGGAGGCTA \\
$\mathrm{Bcl}-2$ & Reverse: GCGCCCAATACGACCAAATC \\
& Forward: GTGAACTGGGGGAGGATTGTG \\
$\mathrm{Bax}$ & Reverse: TTCAGAGACAGCCAGGAGAAATC \\
$\mathrm{Ki}-67$ & Forward: TTTTGCTTCAGGGTTTCATCCA \\
& Reverse: TGCCACTCGGAAAAAGACCTC \\
& Forward: ACGCCTGGTTACTATCAAAAGG \\
\hline
\end{tabular}

Table S2 IC 50 values of DOX and DOX@SUR against MCF-7 and MCF-7/ADR cells after $48 \mathrm{~h}$ of treatment as determined by MTT assay

\begin{tabular}{llll}
\hline IC ${ }_{50}$ values $(\mu \mathrm{g} / \mathrm{mL})$ & & \\
\hline MCF-7 & & MCF-7/ADR & DOX@SUR \\
\cline { 3 - 4 } DOX & DOX@SUR & DOX & 147 \\
\hline 0.35 & 0.44 & 147.6 & 15 \\
\hline
\end{tabular}

Abbreviations: DOX, doxorubicin; SUR, surfactin; DOX@SUR, DOX-loaded surfactin.

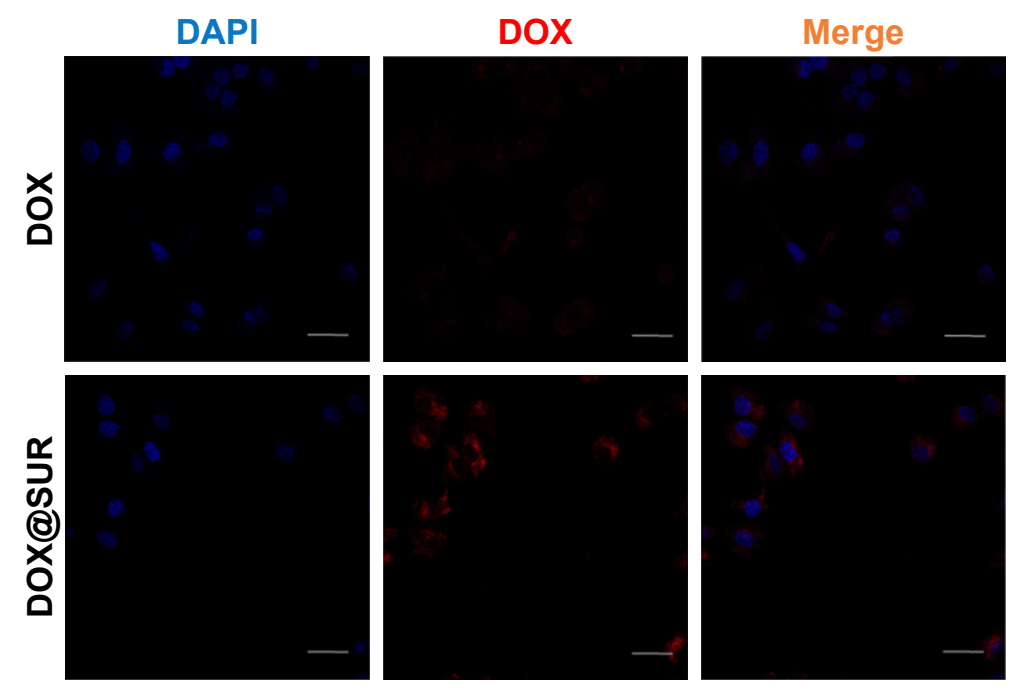

Figure SI Confocal microscopic images of the intracellular DOX localization of MCF-7/ADR cells treated with free DOX or DOX@SUR nanoparticles at a DOX concentration of $5 \mu \mathrm{g} / \mathrm{mL}$ for $8 \mathrm{~h}$.

Note: Scale bar: $25 \mu \mathrm{m}$.

Abbreviations: DAPI, 4,6-diamidino-2-phenylindole; DOX, doxorubicin; SUR, surfactin; DOX@SUR, DOX-loaded SUR. 


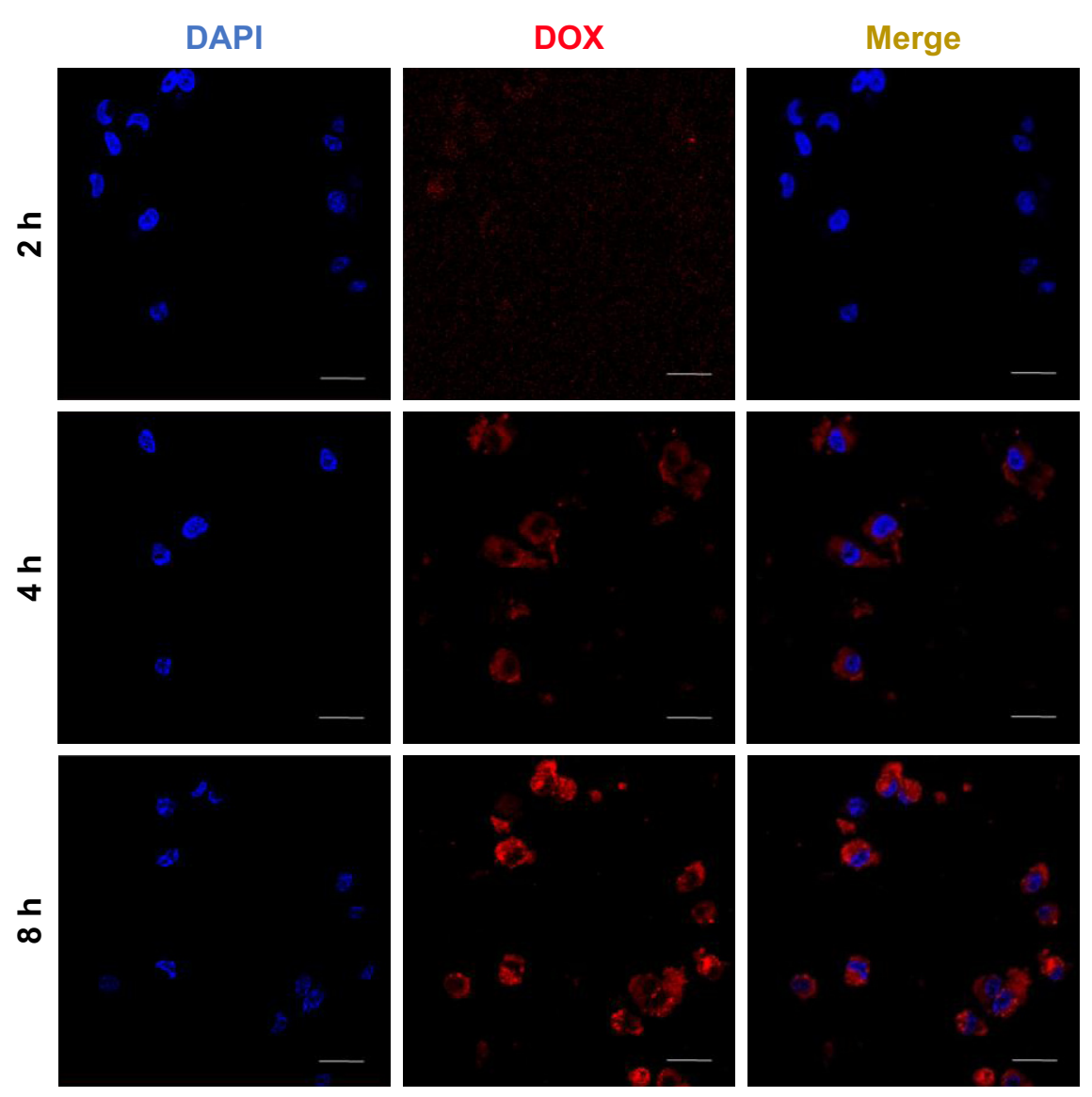

Figure S2 Confocal microscopic images of the intracellular DOX localization of MCF-7/ADR cells treated with DOX@SUR nanoparticles at a DOX concentration of $5 \mu \mathrm{g} / \mathrm{mL}$ for different time courses.

Note: Scale bar: $25 \mu \mathrm{m}$.

Abbreviations: DAPI, 4,6-diamidino-2-phenylindole; DOX, doxorubicin; SUR, surfactin; DOX@SUR, DOX-loaded surfactin.

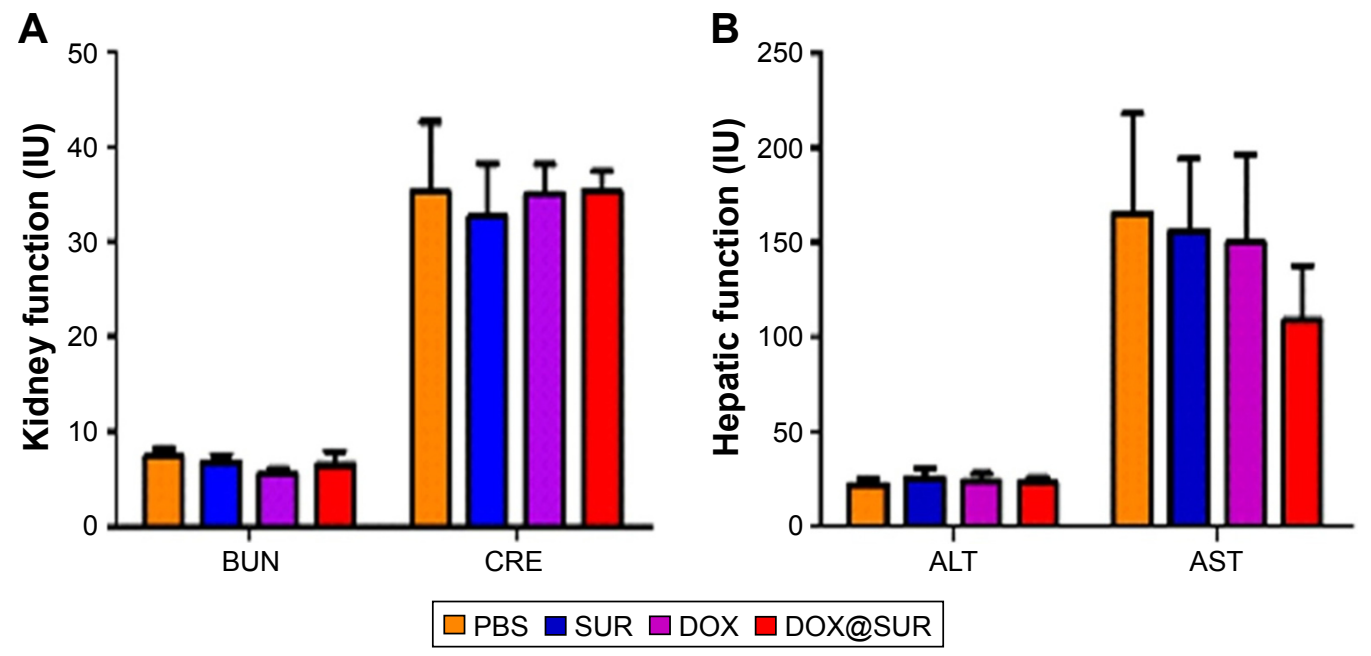

Figure S3 Serological analysis of MCF-7/ADR-bearing nude mice at the end of tumor inhibition experiments, including ALT, AST, BUN and CRE.

Note: Data are represented as mean value $\pm S D(n=6)$.

Abbreviations: ALT, alanine transaminase; AST, aspartate aminotransferase; BUN, blood urea nitrogen; CRE, creatinine; DOX, doxorubicin; SUR, surfactin; DOX@SUR, DOX-loaded surfactin. 


\section{Publish your work in this journal}

The International Journal of Nanomedicine is an international, peerreviewed journal focusing on the application of nanotechnology in diagnostics, therapeutics, and drug delivery systems throughout the biomedical field. This journal is indexed on PubMed Central,

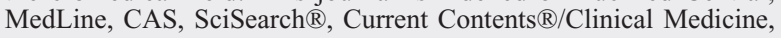

Journal Citation Reports/Science Edition, EMBase, Scopus and the Elsevier Bibliographic databases. The manuscript management system is completely online and includes a very quick and fair peer-review system, which is all easy to use. Visit http://www.dovepress.com/ testimonials.php to read real quotes from published authors.

Submit your manuscript here: http://www.dovepress.com/international-journal-of-nanomedicine-journal 